\title{
What's in Metabolomics for Alcoholic Liver Disease?
}

\author{
Alina M. Suciu ${ }^{1,2}$, Dana A. Crisan ${ }^{3}$, Bogdan D. Procopet ${ }^{1,2}$, Corina I. Radu ${ }^{1,2}$, Carmen Socaciu ${ }^{4}$, Marcel V. Tantau ${ }^{1,2}$, Horia \\ O. Stefanescu ${ }^{2}$, Mircea Grigorescu ${ }^{1}$
}

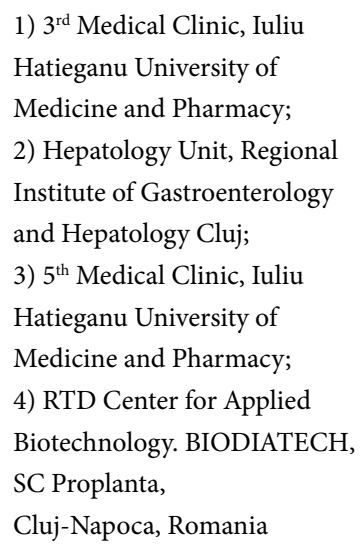

1) $3^{\text {rd }}$ Medical Clinic, Iuliu Hatieganu University of Medicine and Pharmacy; 2) Hepatology Unit, Regional Institute of Gastroenterology and Hepatology Cluj; 3) $5^{\text {th }}$ Medical Clinic, Iuliu Hatieganu University of Medicine and Pharmacy; 4) RTD Center for Applied Biotechnology. BIODIATECH, SC Proplanta, Cluj-Napoca, Romania

\begin{abstract}
Background \& Aims: Current management of alcoholic liver disease (ALD), especially for alcoholic hepatitis $(\mathrm{AH})$ is still driven by liver biopsy. Therefore, the identification of novel and accurate noninvasive biomarkers for the diagnosis and assessment of severity is important. Metabolomics, because it unravels changes closest to the phenotype, may represent the key for novel biomarkers. The aim of this study was to identify and characterize potential metabolomic biomarkers for diagnosis, staging and severity assessment of ALD.

Methods: 30 consecutive ALD patients and 10 healthy controls were included in this proof-of-concept crosssectional study. Baseline assessment consisted in evaluation of Maddrey's Discriminant Function, Model for End-Stage Liver Disease (MELD) and ABIC scores as well as ASH-Test (Fibromax) as a surrogate for the confirmatory diagnosis of AH in suggestive clinical and biologic settings. Additionally, SOP metabolomics and lipidomics were performed from serum samples by liquid chromatography mass-spectrometry analysis. Results: From the 127 and 135 serum/urine candidate metabolites initially identified, only 11/5 metabolites were characteristic for ALD patients. None of them correlated with alcohol intake, and only 5/1 metabolites could differentiate cirrhotic from non-cirrhotic patients. Of those, N-Lauroglycine (NLG) was the best for identifying cirrhosis (100\% sensitivity and 90\% negative predictive value, NPV) and decatrienoic acid (DTEA) was the best for assessing disease severity (evaluated by ABIC score) with $100 \%$ sensitivity and $100 \%$ NPV. Conclusion: Due to their high NPV, NLG and DTEA could be used in conjunction in ALD patients to exclude cirrhosis or a severe disease. If further validated, they could become biomarkers for better management and risk assessment in ALD.
\end{abstract}

Key words: metabolomics - lipidomics - alcoholic liver disease - noninvasive.

Abbreviations: ABIC: age-bilirubin-INR-cholesterol; ALD: alcoholic liver disease; AH: alcoholic hepatitis; AST/ALT: aspartate/alanine aminotransferase; DF: Maddrey's Discriminant Function; DTEA: decatrienoic acid; LC-MS: liquid chromatography-mass spectrometry; LRD: liver related clinical decompensation; MELD: Model for End-Stage Liver Disease; NLG: N-Lauroglycine; NPV: negative predictive value; PPV: positive predictive value; PT: prothrombin time; Se: sensibility; Sp: specificity.

\section{INTRODUCTION}

Received: 15.10.2017 Accepted: 14.12.2017
Alcoholic liver disease (ALD) represents a wide spectrum of liver pathology, beginning with fatty liver, present in almost all heavy alcohol drinkers and mostly asymptomatic, and continuing with progressive fibrosis that eventually leads to cirrhosis $[1,2]$. In the Western world, alcohol is the leading cause of cirrhosis and its complications: portal hypertension, ascites, spontaneous bacterial peritonitis, hepatic encephalopathy, variceal bleeding and hepato-renal syndrome [3]. However, ALD is marked by the spectrum of alcoholic hepatitis (AH), which may develop anytime during the natural history. Alcoholic hepatitis is a clinical syndrome characterized by rapid hepatic decompensation (jaundice, coagulation impairment and encephalopathy) that causes death in up to $50 \%$ of patients in the absence of treatment [4]. Since $20-40 \%$ of alcoholics develop fibrosis and $10-22 \%$ will eventually progress to cirrhosis, of whom 1.5-2\% will develop hepatocellular carcinoma every year [5], it is very important to diagnose and treat early and accurately ALD and alcohol misuse. In none of the stages of ALD are clinical and biological changes characteristic, and the clinical scenario of $\mathrm{AH}$ is very similar to 
the one of severe cirrhotic decompensation of another etiology. In this context, liver biopsy remains the gold standard for diagnosis, mainly for $\mathrm{AH}$, despite its invasiveness and relatively high cost. Therefore, the identification of novel and accurate noninvasive biomarkers for the diagnosis and assessment of severity is of utmost importance.

The metabolome represents the endpoint of the omics cascade and it is also the closest point to the phenotype [7, 8]. Metabolomics is a rapidly evolving field, which identifies characteristic changes in the metabolome associated with any physiological perturbations. The use of metabolomics in ALD represents a powerful means not only to unravel the molecular mechanism of its pathogenesis, but also to identify the earliest biomarkers $[9,10]$. The earliest change in ALD pathogenesis is the accumulation of free fatty acids in the liver. In this context, the majority of new studies are focused on the understanding of ALD pathogenesis by identifying the pathways involved in fatty acid metabolism.

The aim of this proof-of-concept study was to identify and further characterize the potential metabolomic biomarkers for the diagnosis, staging and severity assessment of ALD.

\section{METHODS}

The study was designed as a cross-sectional one, in full accordance with the 2000 review of Human Rights Declaration and was approved by the Ethical Committee of the Cluj-Napoca Regional Institute of Gastroenterology and Hepatology. All participants gave their informed consent prior to inclusion into the study.

\section{Patients}

Consecutive patients previously diagnosed with different stages of ALD, aged 18-80 years and with ongoing alcohol consumption ( $>20 \mathrm{~g} /$ day for women and $>40 \mathrm{~g} /$ day for men) were included. Patients with cirrhosis have been also previously diagnosed based on unequivocal clinical, biological, imaging and endoscopic features.

Additionally, consecutive subjects without liver disease and without significant alcohol intake $(<20 \mathrm{~g} /$ day for women and $<40 \mathrm{~g} /$ day for men) were included and were considered as control group.

\section{Baseline assessment}

Fasting serum, plasma and urine samples were collected from all participants for metabolomic analysis (see below).

Patients with ALD underwent in the same day a full laboratory work-up assessing liver function tests, coagulation, platelets count, serum lipids and glucose. ASH-Test (BioPredictive, France) was performed in all patients in order to evaluate the level of necro-inflammation and was used as a surrogate for $\mathrm{AH}$ diagnosis in suggestive clinico-biologic settings. Demographic and anthropometric data were noted. Clinical examination, abdominal ultrasound and liver stiffness measurements were performed.

For the evaluation of the severity of ALD the following scores were calculated:

- Maddrey's Discriminant Function (DF), which depends on bilirubin levels and PT: a cut-off value $\geq 32$ is correlated with a more severe outcome, lack of response to corticotherapy and death in almost $50 \%$ of the patients [11];

- Model for End-Stage Liver Disease (MELD), which uses bilirubin, INR and creatinine to assess liver disease severity and predict mortality [12];

- ABIC score, which integrates age, bilirubin level, INR, creatinine, being able to predict 90 days mortality [14]. ABIC allows mortality risk stratification into low, moderate or high.

\section{Standard Operation Procedure (SOP) Metabolomics Blood sample preparation}

The serum samples were diluted (1:5) with methanol, vortexed, ultrasonicated at $4^{\circ} \mathrm{C}$ for 5 minutes and centrifuged at $15000 \mathrm{~g}$ for 15 minutes to remove particulates and proteins by precipitation. The supernatant was collected, filtered through $0.2 \mu \mathrm{m}$ filters and kept in the deep freezer until analysis.

\section{Liquid chromatography mass spectrometry analysis}

Aliquots of $5 \mu \mathrm{l}$ of each sample were subjected to chromatography on a Bruker DaltonicsMaXis Impact device with a Thermo Scientific HPLC UltiMate 3000 system with a quaternary pump delivery system DionexUltiMate and MS detection, on C18 reverse-phase column [ $5 \mu \mathrm{m}, 2.1 \mathrm{x} 100 \mathrm{~mm}$ ], (Acclaim, Dionex) maintained at $40^{\circ} \mathrm{C}$.

Mobile phase: A - water containing $0.1 \%$ formic acid; B acetonitrile containing $0.1 \%$ formic acid. Gradient: 95\% A:5\% B with linear gradient to $85 \%$ A: $15 \%$ B from 0 to $3 \mathrm{~min}$, followed by linear gradient to $50 \%$ A: $50 \% \mathrm{~B}$ at $6 \mathrm{~min}$, linear gradient to $5 \% \mathrm{~A}: 95 \% \mathrm{~B}$ at $9 \mathrm{~min}$, isocratic on $5 \% \mathrm{~A}: 95 \% \mathrm{~B}$ for $6 \mathrm{~min}$ and then returned to the initial condition $95 \% \mathrm{~A}: 5 \% \mathrm{~B}$ at 15.1 $\mathrm{min}$ for $5 \mathrm{~min}$. Flow rate, $0.500 \mathrm{ml} / \mathrm{min}$.

Mass spectrometry was performed on a Bruker Daltonics MaXis Impact Q-TOF operating in positive ion mode. The mass range was set between $50-1000 \mathrm{~m} / \mathrm{z}$. The nebulizing gas pressure was set at $2.8 \mathrm{bar}$, the drying gas flow at $12 \mathrm{~L} / \mathrm{min}$, the drying gas temperature at $300^{\circ} \mathrm{C}$. Before each chromatographic run, a calibrant solution of sodium formate was injected.

The control of the instrument and data processing were done using TofControl 3.2 and Data Analysis 4.2 (Bruker Daltonics).

\section{SOP lipidomics}

\section{Blood sample preparation (lipid extraction)}

Lipids were extracted from $0.1 \mathrm{ml}$ serum diluted with $0.2 \mathrm{ml}$ methanol, then vortexed for $20 \mathrm{~s}$, next $1.66 \mathrm{ml}$ chloroform was added and vortexed for $20 \mathrm{~s}$, and an aditional of $0.1 \mathrm{ml}$ water was added to induce phase separation. The samples obtained were vortexed for $20 \mathrm{~s}$, then were subjected to centrifugation at $8000 \mathrm{rpm}$ for $10 \mathrm{~min}$, then the lipid phase was collected and evaporated. Samples were reconstituted in $500 \mu \mathrm{l}$ of acetonitrile/isopropylic acid/water (65:30:5) volume.

\section{Liquid chromatography mass spectrometry analysis}

Aliquots of $5 \mu \mathrm{l}$ of each sample were subjected to chromatography on a Bruker DaltonicsMaXis Impact device with a Thermo Scientific HPLC UltiMate 3000 system with a quaternary pump delivery system DionexUltiMate and MS detection, on C18 reverse-phase column [5 $\mathrm{m}, 2.1 \times 100 \mathrm{~mm}$ ], (Acclaim, Dionex) maintained at $55^{\circ} \mathrm{C}$.

Mobile phase: A - water: acetonitrile (60:40) containing $0.1 \%$ formic acid and $10 \mathrm{mM}$ ammonium formate; $\mathrm{B}$ - isopropyl 
alcohol: acetonitrile (90:10) containing $0.1 \%$ formic acid and $10 \mathrm{mM}$ ammonium formate. Gradient: $75 \%$ A: $25 \%$ B followed by linear gradient to $50 \%$ A: $50 \%$ B at 4 min, linear gradient to $3 \% \mathrm{~A}: 97 \% \mathrm{~B}$ at $19 \mathrm{~min}$, isocratic on $3 \% \mathrm{~A}: 97 \% \mathrm{~B}$ for $4 \mathrm{~min}$ and then returned to the initial condition $75 \%$ A: $25 \%$ B at 24 $\mathrm{min}$ for $4 \mathrm{~min}$. Flow rate, $0.260 \mathrm{ml} / \mathrm{min}$.

Mass spectrometry was performed on a Bruker Daltonics MaXis Impact Q-TOF operating in positive ion mode. The mass range was set between $50-1000 \mathrm{~m} / \mathrm{z}$. The nebulizing gas pressure was set at $2.8 \mathrm{bar}$, the drying gas flow at $12 \mathrm{~L} / \mathrm{min}$, the drying gas temperature at $300^{\circ} \mathrm{C}$. Before each chromatographic run, a calibrant solution of sodium formate was injected.

The control of the instrument and the data processing were completed using Tof Control 3.2 and Data Analysis 4.2 (Bruker Daltonics).

\section{RESULTS}

\section{Baseline characteristics of the patients}

Ten controls and 30 patients with ALD were enrolled. Of them, 17 were known to have cirrhosis and 7 had previous liver related clinical decompensation (LRD) events. Of the 30 ALD patients at inclusion, 16 patients (of which 12 were known cirrhotics) had an ASH-Test $\geq 0.18$, indicating at least minimal activity.

Baseline patient characteristics are shown in Table I.

\section{Identification of the candidate metabolites}

Using liquid chromatography-mass spectrometry (LC-MS) of both serum and urine samples of the entire cohort of patients with ALD and compared with those of healthy controls (Fig. 1) we identified 127 serum candidate metabolites for ALD
Table I. Baseline ALD patients' characteristics

\begin{tabular}{ll}
\hline Variables & Value \\
\hline Age (years) & $53.2(27-74)$ \\
Male/Female & $19 / 11$ \\
Height $(\mathrm{cm})$ & $164.249 \pm 6.0412$ \\
Weight $(\mathrm{kg})$ & $69.561 \pm 14.9096$ \\
ALT $(\mathrm{IU} / \mathrm{ml})$ & $67.43(17-260)$ \\
AST $(\mathrm{IU} / \mathrm{ml})$ & $47.8(7-169)$ \\
Bilirubin (mg/dl) & $8.25(0.3-68.43)$ \\
Cholesterol (mg/dl) & $191.03(89-394)$ \\
Triglycerides (mg/dl) & $115.96(3-228)$ \\
GGT (IU/ml) & $242.86(25-2229)$ \\
Glucose (mg/dl) & $130.89(66-405)$ \\
Platelets & $162,493(27,000-367,000)$ \\
Alcohol consumption (g/day) & $109.7(31.8-320)$ \\
Cirrhosis n (\%) & $17(56.66 \%)$ \\
With decompensation & $7(23.33 \%)$ \\
MELD score & $13.46(6-29)$ \\
Maddrey DF & $28(8-36)$ \\
ABIC score & $26(6-29)$ \\
\hline &
\end{tabular}

patients and 150 for controls. From the urine we identified 135 candidate metabolites for the ALD cohort and 129 for controls.

By using the Scores system, 35 major metabolites could be further identified (Fig. 2).

Further more, using the Loading system, based on $\mathrm{m} / \mathrm{z}$ analysis (Fig. 3), we identified 11 serum and 5 urine metabolites that were significantly different in patients with ALD compared with controls. The serum and urine metabolites in patients with
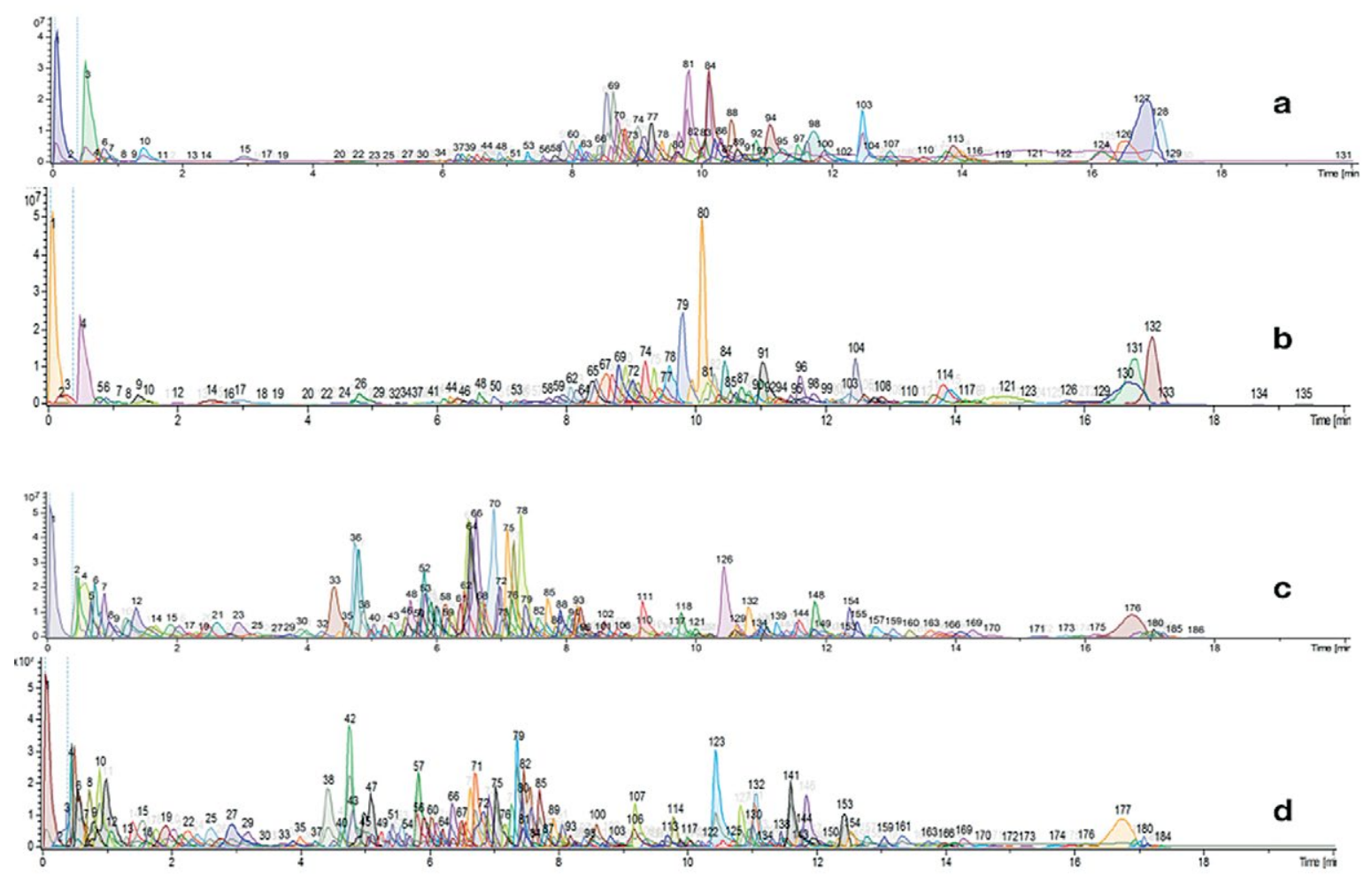

Fig. 1. Skyline graph demonstrating the metabolites identified in the serum of patients with ALD (a) and healthy controls (b) as well as in the urine of patients (c) and controls (d). 

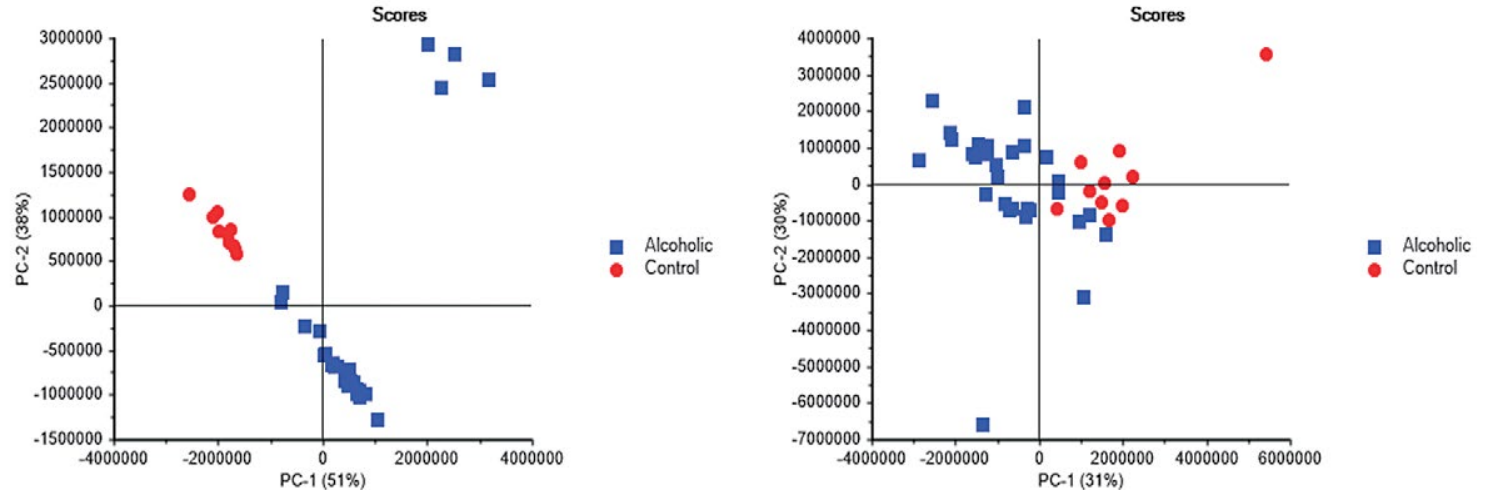

Fig. 2. Scores system-identification of 35 major metabolites from serum and urine in ALD patients vs controls
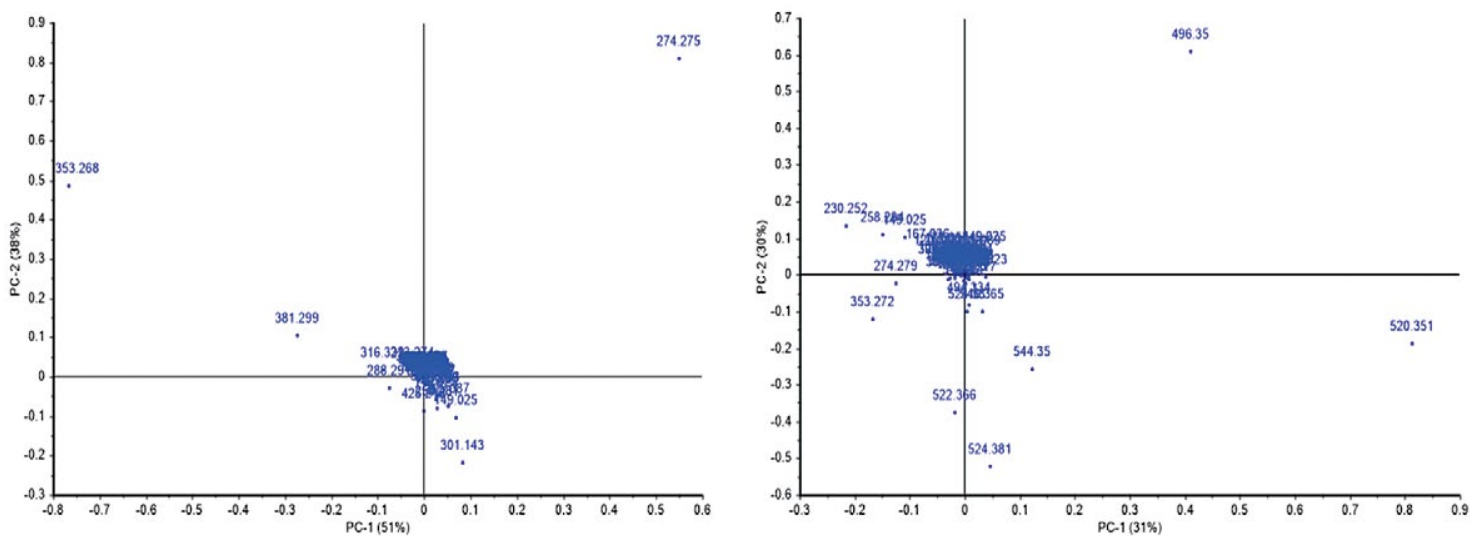

Fig. 3. Loadings system identifying the specific metabolites in serum and urine of ALD patients

ALD identified using sequential metabolomic techniques are shown in Table II.

\section{Characterization of the selected metabolites}

The signal areas of these 11 serum and 5 urine metabolites were further analyzed in patients with ALD. None of these metabolites was correlated with the amount of alcohol consumption (units/day).

Only 5 serum (sM2, 3, 5, 10 and 11$)$ and 1 urine (uM3) metabolites were able to further differentiate cirrhotic from non-cirrhotic patients with ALD. These 6 metabolites were chosen for further deeper analysis as represented in Fig. 4.

Table II. Serum and urine metabolites in patients with ALD identified using sequential metabolomic techniques

\begin{tabular}{|c|c|c|c|c|c|}
\hline \multicolumn{3}{|c|}{ Serum Metabolites } & \multicolumn{3}{|c|}{ Urine Metabolites } \\
\hline Code & $\mathrm{M} / \mathrm{Z}$ Value & Common Name & Code & M/Z Value & Common Name \\
\hline sM1 & 149.022 & 2-Hydroxyglutarate & uM1 & 274.275 & Glutaconylcarnitine \\
\hline sM2 & 167.054 & Decatrienoic acid & uM2 & 288.291 & L-Octanoylcarnitine \\
\hline sM3 & 177.0545 & Ascorbic acid & uM3 & 301.142 & 2-Methoxyestrone \\
\hline sM4 & 230.2486 & Butenyl carnitine & uM4 & 353.266 & $\begin{array}{c}\text { MG(18:3/0:0/0:0) } \\
\text { MG(0:0/18:3/0:0) } \\
\text { Prostaglandin D2/E2 }\end{array}$ \\
\hline sM5 & 258.2797 & $\mathrm{~N}$-Lauroylglycine & uM5 & 381.298 & $\begin{array}{l}\operatorname{MG}(20: 3 / 0: 0 / 0: 0) \\
\operatorname{MG}(0: 0 / 20: 3 / 0: 0)\end{array}$ \\
\hline sM6 & 353.272 & $\begin{array}{l}\text { Prostaglandin E2/D2/H2 } \\
\text { MG (0:0/18:3/0:0) }\end{array}$ & & & \\
\hline sM7 & 496.3407 & $\begin{array}{l}\mathrm{PE}(\mathrm{O}-20: 0 / 0: 0) \\
\operatorname{PS}(16: 1(9 \mathrm{Z}) / 0: 0)\end{array}$ & & & \\
\hline sM8 & 520.3407 & LysoPC $(18: 2(9 \mathrm{Z}, 12 \mathrm{Z}) / 0: 0)$ & & & \\
\hline sM9 & 522.3569 & LysoPC (18:1(9Z)/0:0) & & & \\
\hline sM10 & 524.3721 & $\begin{array}{l}\text { Isom.LPC (16:0/2:0), } \\
\text { LysoPC (0:0/18:0) }\end{array}$ & & & \\
\hline sM11 & 544.341 & LysoPC (20:4/0:0) & & & \\
\hline
\end{tabular}


Table III. Signal areas of 5 serum and lurine metabolites in cirrhotic vs. non-cirrhotic patients with ALD and their diagnostic performance for cirrhosis

\begin{tabular}{|c|c|c|c|c|c|}
\hline & \multicolumn{3}{|c|}{ Signal areas } & \multicolumn{2}{|c|}{ Ability to diagnose cirrhosis } \\
\hline & Cirrhosis & Non-cirrhosis & $\mathrm{p}$ & AUROC & $95 \%$ CI \\
\hline sM2 & $\begin{array}{c}684060.313 \\
( \pm 150311.430)\end{array}$ & $\begin{array}{c}544880.286 \\
( \pm 165017.511)\end{array}$ & 0.022 & 0.763 & $0.574-0.898$ \\
\hline sM3 & $\begin{array}{c}902229.563 \\
( \pm 126759.965)\end{array}$ & $\begin{array}{c}806594.357 \\
( \pm 109529.537)\end{array}$ & 0.037 & 0.759 & $0.569-0.895$ \\
\hline sM5 & $\begin{array}{c}2676623.06 \\
( \pm 320180.649)\end{array}$ & $\begin{array}{c}2401624.71 \\
( \pm 253205.174)\end{array}$ & 0.015 & $0.835^{\star}$ & $0.655-0.944$ \\
\hline sM10 & $\begin{array}{c}350893.875 \\
( \pm 133338.296)\end{array}$ & $\begin{array}{c}545040.714 \\
( \pm 192818.251)\end{array}$ & 0.003 & 0.728 & $0.535-0.873$ \\
\hline sM11 & $\begin{array}{c}790748.438 \\
( \pm 493497.928)\end{array}$ & $\begin{array}{c}1633634.14 \\
( \pm 755245.193)\end{array}$ & 0.001 & 0.728 & $0.535-0.873$ \\
\hline uM3 & $\begin{array}{c}288674.875 \\
( \pm 16968.302)\end{array}$ & $\begin{array}{c}275233.214 \\
( \pm 24205.543)\end{array}$ & 0.08 & 0.656 & $0.456-0.858$ \\
\hline
\end{tabular}

${ }^{*} \mathrm{p}$ (deLong test) $=0.05$ (vs. sM2 and sM3) and 0.03 (vs. sM10 and sM11), respectively

\section{Ability to diagnose cirrhosis}

Analyzing only the ALD patients, all the selected serum metabolites had significantly different mean signal areas in patients with cirrhosis as compared with those without, while uM3 lost the significance. The diagnostic performance for cirrhosis was acceptable for all metabolites, but sM5 was significantly better than all the others (Table III).

Based on these data, sM5 (N-Lauroglycine, NLG) was chosen as the best metabolite for prediction of cirrhosis in patients with ALD. For a cut-off value of the signal area of 2478287, the sensitivity (Se) was 1 and specificity (Sp) 0.62, the positive predictive value (PPV) was $75 \%$ and negative predicted value (NPV) 90\%, the positive and negative likelihood ratio (LR) were 2.62 and 0.09 , respectively. Using this value to assess the diagnostic accuracy, 24/30 (80\%) patients were correctly classified (chi-square $=11.317, \mathrm{p}=0.001$ ).

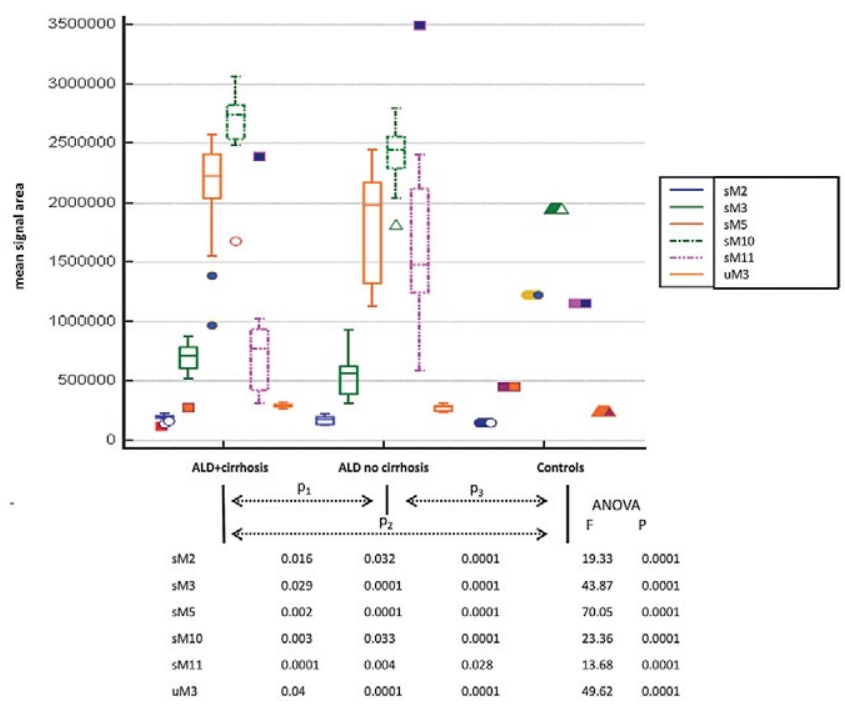

Fig. 4. Metabolites characterization. Boxplots representing signal areas for 5 serum and lurine metabolites in cirrhotic, non cirrhotic patients with ALD and healthy controls, as depicted by ANOVA test with Bonferroni post-hoc analysis. $\mathrm{p} 1, \mathrm{p} 2$ and $\mathrm{p} 3$ represent the $\mathrm{p}$ values comparing mean signal areas between cirrhotic-non cirrhotic patients, cirrhotics-controls and non-cirrhotics-controls, respectively; $\mathrm{P}$ and $\mathrm{F}$ represent the values of overall ANOVA test for each metabolite).
Table IV. Correlation of serum metabolites with ALD severity indexes; the urine metabolite was not correlated with either of these scores.

\begin{tabular}{cccc}
\hline & & Maddrey score & ABIC \\
\hline \multirow{2}{*}{ sM2 } & rho & 0.529 & 0.679 \\
& $\mathrm{p}$ & 0.003 & 0.0001 \\
sM3 & rho & 0.413 & 0.561 \\
& $\mathrm{p}$ & 0.01 & 0.001 \\
sM5 & rho & 0.557 & 0.560 \\
& $\mathrm{p}$ & 0.001 & 0.001 \\
sM10 & rho & -0.569 & -0.538 \\
& $\mathrm{p}$ & 0.001 & 0.002 \\
sM11 & rho & -0.530 & -0.616 \\
& $\mathrm{p}$ & 0.003 & 0.0001 \\
\hline
\end{tabular}

\section{Assessment of disease severity}

All the five serum metabolites were well correlated with Maddrey and ABIC scores, as the most widely used in clinical practice to assess ALD severity and prognosis (Table IV).

Based on these data, sM2 and sM5 seem to be the best candidates to be further used to assess ALD severity. Both metabolites had similar AUROC to predict Maddrey score $\geq 32$ (0.799 (95\%CI: 0.613-0.922) vs. 0.837 (95\%CI: 0.681-0.937); $\mathrm{p}$ (de Long test $)=0.12$ and, respectively ABIC score $\geq 6.71(0.844$ (95\%CI: 0.666-0.950) vs. 0.867 (95\%CI:0.693-0.962); p (de Long test $)=0.73$.

As for the ABIC score $>9$ (associated with severe disease and bad prognosis), the AUROC for sM2 was significantly higher than the one for sM5: 0.946 (95\%CI: 0.863-1.000) vs. 0.884 (95\%CI: 0.714-0.971), p (de Long test) $=0.05$. Based on these findings, sM2 (decatrienoic acid, DTEA) was considered as being the best serum metabolite to assess the disease severity (Table V).

\section{DISCUSSION}

The purpose of this study was to identify and characterize new metabolomic biomarkers for the diagnosis, staging and severity assessment of ALD, as a noninvasive alternative to 
Table V. Association between the decatrienoic acid (DTEA) area and prognostic scores.

\begin{tabular}{lccc}
\hline & Maddrey $>32$ & ABIC $\geq 6.71$ & ABIC $>9$ \\
\hline Cut-off value & $>624000$ & $>624000$ & $>819000$ \\
Sensitivity & 0.86 & 0.79 & 1 \\
Specificity & 0.75 & 0.75 & 0.93 \\
PPV & $75.00 \%$ & $80 \%$ & 50 \\
NPV & $85.00 \%$ & $78.60 \%$ & 100 \\
+LR & 3.42 & 3.73 & 14 \\
-LR & 0.19 & 0.25 & 0 \\
Diagnostic accuracy & $80 \%$ & $79.31 \%$ & $93.33 \%$ \\
Correctly class pts & $24 / 30$ & $23 / 30$ & $28 / 30$ \\
\hline
\end{tabular}

LR: likehood ratio; NPV: negative predictive value; PPV: positive predictive value.

liver biopsy. Metabolomics is the last and rapidly expanding omic technique that explores the wide spectrum of metabolic pathways products. Theoretically, these products should be the first to change when a certain pathway is interfered by a specific condition, thus metabolomics appears to be the path to follow when trying to identify sensitive and specific biomarkers for early diagnosis. By using different methods to purify the metabolites' signature obtained from patients with ALD, we identified 5 serum and 1 urine metabolites, of which one (NLG) seemed accurate for identifying cirrhotic patients and another (DTEA) appeared to be more appropriate to select patients with severe disease.

Multiple studies have analyzed the metabolomics profile for other hepatic diseases such as nonalcoholic fatty liver disease (NAFLD), viral hepatitis and hepatocellular carcinoma [24-33], trying to identify possible biomarkers for disease progression. In NAFLD, elevated hepatic concentrations of various lysophosphatidylcholine (LPC), lysophosphatidylethanolamine (LPE) and phosphatidylcholine (PC) species have been reported for human steatotic vs. nonsteatotic livers $[24,30]$. Three studies reported elevated bile salts in the liver [24] that spilled over to elevated bile acids in serum and plasma $[25,26]$. Regarding HCV infection, a metabolomic comparison of $\mathrm{HCV}$-infected hepatocytes revealed small but significant increases in alanine, tyrosine and adenosine [27, $30,32]$. The metabolomic changes in hepatocellular carcinoma tend to point to increased fatty acid $\beta$-oxidation, with elevated acetate and 2-oxoglutarate (precursor of carnitine) and reduced free fatty acids, carnitine and carnitine esters [30,31]. Further studies are required to validate these molecules as biomarkers for hepatocellular carcinoma.

Until now, only a few studies have focused on the metabolomic assessment of ALD [15-19]. The data available are heterogeneous, since the design of these studies differed substantially. Most of the studies identified protein metabolites associated with ALD in experimental [18] or clinical settings, either from serum [15] or urine [16] of drinking men. Overall, these studies found 19 metabolites associated with alcohol intake, most derived from the protein metabolism, and some of them, possible biomarker candidates of alcohol-induced liver injury.
Since the lipid metabolism appears to be the first dysregulated in the development of ALD, it seems rational to search for lipid metabolites as early biomarkers. In this respect, Li et al. [19] found in a murine model that metabolites of phosphatidyl choline, sphyngomielin as well as some aminoacids were associated with the development of hepatocellular carcinoma in ALD [19]. Our group previously identified an isoform of LPC to be a good predictor of liver related decompensation and death in patients with severe $\mathrm{AH}$ [33]. Similarly, all the relevant metabolites identified by this study using an untargeted approach are also related with the lipid pathway, demonstrating the importance of this pathway in the development and progression of ALD. There are multiple possible host or environmental factors that might interfere with the pathogenesis of ALD: drinking patterns, diet, microbiota composition. However, our study was not designed to evaluate neither of these factors, except the amount of alcohol intake and none of the identified metabolites were directly related with alcohol consumption.

The differential diagnosis between $\mathrm{AH}$ and cirrhotic decompensation is often difficult, because of similar clinical, biological and imaging aspects [20-22]. In this respect, the metabolite identified in our study (NLG), due to the high NPV, appears useful to select patients without cirrhosis. On the other hand, severity assessment in ALD and AH is extremely important and the performance of currently available systems (DF, MELD, ABIC) needs to be improved. In this respect, we identified DTEA as a possible biomarker to exclude severe $\mathrm{AH}$.

The relevance of our data is hampered by the study limitations. There are some intrinsic limitations: the small number of patients, which is however acceptable for a proofof-concept approach; the lack of biopsy-proven ALD and AH; the cross-sectional design and also the fact that the response to therapy was not assessed. Besides that, there are the limitations of the metabolomic approach, especially the untargeted one: lack of well established and standardized methods or procedures, metabolite identification difficult and time consuming, potentially thousands of compounds can match a given parent ion mass or a given atomic composition [34].

Despite these drawbacks, metabolomics seems to offer the premises of identifying an "ideal" biomarker for ALD: specific, easy to use, widely available and also with a low cost. Our study was not designed to prove any of these facts, but opened the gate of opportunity for a closer look into metabolomics' applications in ALD. Until now, we managed to identify two serum metabolites with a good diagnostic accuracy and high NPV that could be used in conjunction in patients with ALD for better stratification: NLG to exclude the presence of cirrhosis and DTEA to exclude a severe disease. Nevertheless, all these findings need to be further addressed in larger, prospective clinical trials, with relevant clinical and therapeutic end-points.

\section{CONCLUSION}

Based on this proof of concept study, metabolomics appears to offer both the opportunity and the means for better management and risk assessment of patients with ALD. 
Conflicts of interest: No conflict to declare.

Authors' contribution: A.S. drafted the manuscript; H.S. critically revised and completed the manuscript; A.S., D.C., C.R., B.P. and H.S. evaluated the patients and revised the manuscript; CS performed metabolomic analysis; M.T. and M.G. approved and coordinated the study and revised the manuscript.

Acknowledgement: This study was financially supported by a grant from the Romanian Ministry of Education and Research (UEFISCDI): (PN-II-RU-TE-2014-4-0709, PN-II-RU-TE-2014-4-0356).

\section{REFERENCES}

1. Miller AM, Horiguchi N, Jeong WI, Radaeva S, Gao B. Molecular mechanisms of alcoholic liver disease: innate immunity and cytokines. Alcohol Clin Exp Res 2011;35:787-793. doi:10.1111/j.15300277.2010.01399.x

2. Mandrekar P. Epigenetic regulation in alcoholic liver disease. World J Gastroenterol 2011;17:2456-2464. doi:10.3748/wjg.v17.i20.2456

3. Teli MR, Day CP, Burt AD, Bennett MK, James OF. Determinants of progression to cirrhosis or fibrosis in pure alcoholic fatty liver. Lancet 1995;346:987-990.

4. Poynard T, Mathurin P, Lai CL, et al. A comparison of fibrosis progression in chronic liver diseases. J Hepatol 2003;38:257-265. doi:10.1016/S0168-8278(02)00413-0

5. Peery AF, Dellon ES, Lund J, et al. Burden of gastrointestinal disease in the United States: 2012 update. Gastroenterology 2012;143:1179-1187. doi:10.1053/j.gastro.2012.08.002

6. Grant BF. Barriers to alcoholism treatment: Reasons for not seeking treatment in a general population sample. J Stud Alcohol 1997;58:365371. doi:10.15288/jsa.1997.58.365

7. Nishiumi S, Suzuki M, Kobayashi T, Matsubara A, Azuma T, Yoshida M. Metabolomics for biomarker discovery in gastroenterological cancer. Metabolites 2014;4:547-571. doi:10.3390/metabo4030547

8. Rieder F, Kurada S, Grove D, et al. A Distinct Colon-Derived Breath Metabolome is Associated with Inflammatory Bowel Disease, but not its Complications. Clin Transl Gastroenterol 2016;7:e201. doi:10.1038/ ctg.2016.57

9. Griffin JL, Nicholls AW. Metabolomics as a functional genomic tool for understanding lipid dysfunction in diabetes, obesity and related disorders. Pharmacogenomics 2006;7:1095-1107. doi:10.2217/14622416.7.7.1095

10. Lelliott CJ, Lopez M, Curtis RK, et al. Transcript and metabolite analysis of the effects of tamoxifen in rat liver reveals inhibition of fatty acid synthesis in the presence of hepatic steatosis. Faseb J 2005;19:1108-1119. doi:10.1096/fj.04-3196com

11. Maddrey WC, Boitnott JK, Bedine MS, Weber FL Jr, Mezey E, White RI Jr. Corticosteroid therapy of alcoholic hepatitis. Gastroenterology 1978;75:193-199.

12. Gholam PM. Prognosis and Prognostic Scoring Models for Alcoholic Liver Disease and Acute Alcoholic Hepatitis. Clin Liver Dis 2016;20:491497. doi:10.1016/j.cld.2016.02.007

13. Dominguez M, Rincón D, Abraldes JG, et al. A new scoring system for prognostic stratification of patients with alcoholic hepatitis. Am J Gastroenterol 2008;103:2747-2756. doi:10.1111/j.15720241.2008.02104.x
14. Yu M, Zhu Y, Cong Q, Wu C. Metabonomics Research Progress on Liver Diseases. Can J Gastroenterol Hepatol 2017;2017:8467192. doi:10.1155/2017/8467192

15. Harada S, Takebayashi T, Kurihara A, et al. Metabolomic profiling reveals novel biomarkers of alcohol intake and alcohol-induced liver injury in community-dwelling men. Environ Health Prev Med 2016;21:18-26. doi:10.1007/s12199-015-0494-y

16. Chang HS, Bai HC, Wang M. The urine metabolomics research of patients with alcoholic liver disease. Heilongjiang Medicine and Pharmacy 2013;5:44-45.

17. Nahon $\mathrm{P}$, Amathieu R, Triba MN, et al. Identification of serum proton NMR metabolomic fingerprints associated with hepatocellular carcinoma in patients with alcoholic cirrhosis. Clin Cancer Res 2012;18:6714-6722. doi:10.1158/1078-0432.CCR-12-1099

18. Manna SK, Thompson MD, Gonzalez FJ. Application of mass spectrometry-based metabolomics in identification of early noninvasive biomarkers of alcohol-induced liver disease using mouse model. Adv Exp Med Biol 2015;815:217-238. doi:10.1007/978-3-319-09614-8_13

19. Li S, Liu H, Jin Y, Lin S, Cai Z, Jiang Y. Metabolomics study of alcoholinduced liver injury and hepatocellular carcinoma xenografts in mice. J Chromatogr B Analyt Technol Biomed Life Sci 2011;879:2369-2375. doi:10.1016/j.jchromb.2011.06.018

20. Dhanda AD, Collins PL, McCune CA. Is liver biopsy necessary in the management of alcoholic hepatitis? World J Gastroenterol 2013;19:7825-7829. doi:10.3748/wjg.v19.i44.7825

21. Stickel F, Datz C, Hampe J, Bataller R. Pathophysiology and Management of Alcoholic Liver Disease: Update 2016. Gut Liver 2017;11:173-188. doi:10.5009/gnl16477

22. Hernaez R, Solà E, Moreau R, Ginès P. Acute-on-chronic liver failure: an update. Gut 2017;66:541-553. doi:10.1136/gutjnl-2016-312670

23. Rachakonda V, Gabbert C, Raina A, et al. Serum metabolomic profiling in acute alcoholic hepatitis identifies multiple dysregulated pathways. PLoS One 2014;9:e113860. doi:10.1371/journal.pone.0113860

24. Garcia-Canaveras JC, Donato MT, Castell JV, Lahoz A. A comprehensive untargeted metabonomic analysis of human steatotic liver tissue by RP and HILIC chromatography coupled to mass spectrometry reveals important metabolic alterations. J Proteome Res 2011;10:4825-4834. doi: $10.1021 /$ pr200629p

25. Kalhan SC, Guo L, Edmison J, et al. Plasma metabolomic profile in nonalcoholic fatty liver disease. Metabolism 2011;60:404-413. doi:10.1016/j.metabol.2010.03.006

26. Barr J, Vazquez-Chantada M, Alonso C, et al. Liquid chromatographymass spectrometry-based parallel metabolic profiling of human and mouse model serum reveals putative biomarkers associated with the progression of nonalcoholic fatty liver disease. J Proteome Res 2010;9:4501-4512. doi:10.1021/pr1002593

27. Roe B, Kensicki E, Mohney R, Hall WW. Metabolomic profile of hepatitis C virus-infected hepatocytes. PLoS One 2011;6:e23641. doi:10.1371/ journal.pone.0023641

28. Di Poto C, Ferrarini A, Zhao Y, et al. Metabolomic Characterization of Hepatocellular Carcinoma in Patients with Liver Cirrhosis for Biomarker Discovery. Cancer Epidemiol Biomarkers Prev 2017;26:675683. doi:10.1158/1055-9965.EPI-16-0366

29. Crisan D, Radu C, Suciu A, et al. Metabolomics for genomics: the role of vitamin $\mathrm{D}$ in nonalcoholic fatty liver disease. J Gastrointestin Liver Dis 2015;24:394-395.

30. Beyoğlu D, Idle JR. The metabolomic window into hepatobiliary disease. J Hepatol 2013;59:842-858. doi:10.1016/j.jhep.2013.05.030 
31. Gao R, Cheng J, Fan C, et al. Serum Metabolomics to Identify the Liver Disease-Specific Biomarkers for the Progression of Hepatitis to Hepatocellular Carcinoma. Sci Rep 2015;5:18175. doi:10.1038/srep18175

32. Sarfaraz MO, Myers RP, Coffin CS, et al. A quantitative metabolomics profiling approach for the noninvasive assessment of liver histology in patients with chronic hepatitis C. Clin Transl Med 2016;5:33. doi:10.1186/s40169-016-0109-2
33. Stefanescu H, Suciu A, Romanciuc F, et al. Lyso-phosphatidylcholine: A potential metabolomic biomarker for alcoholic liver disease? Hepatology 2016;64:678-679. doi:10.1002/hep.28630

34. Scalbert A, Brennan L, Fiehn O, et al. Mass-spectrometry-based metabolomics: limitations and recommendations for future progress with particular focus on nutrition research. Metabolomics 2009;5:435458. doi:10.1007/s11306-009-0168-0 